\title{
CURATIVO DE CATETER VENOSO CENTRAL EM PACIENTES RENAIS: CONHECIMENTO E VALOR ATRIBUÍDO PELA EQUIPE DE ENFERMAGEM
}

\author{
Eliane Cristina Sanches Maziero¹, Elaine Drehmer de Almeida Cruz²
}

\begin{abstract}
RESUMO: Pesquisa quantitativa, descritiva e exploratória que teve por objetivo investigar o conhecimento, a adesão e o valor atribuído pela equipe de enfermagem de uma clínica de doenças renais ao protocolo de curativo de cateter venoso central em pacientes hemodializados. Os dados foram coletados por observação sistemática não participante e entrevista individual. A análise foi direcionada pelas dimensões do modelo de crenças em saúde. Os resultados evidenciaram que os sujeitos atribuem valor à suscetibilidade dos pacientes renais usuários de cateter venoso central e em tratamento hemodialítico a desenvolverem infecção, bem como a severidade desses agravos. Reconhecem os benefícios da prática assistencial de qualidade para a sua prevenção. Lacunas entre o conhecimento e a adesão ao protocolo foram observadas, sendo as condições estruturais desfavoráveis identificadas como principais barreiras. Embora os trabalhadores conheçam e atribuam valor ao protocolo institucional de curativo, reconhecendo sua importância na prevenção de infecções, são necessárias estratégias administrativas e educacionais para maior aproximação entre a teoria e a prática assistencial.
\end{abstract}

PALAVRAS-CHAVE: Cateterismo venoso central; Curativos; Hemodiálise.

\section{CENTRAL VENOUS DRESSING IN RENAL PATIENTS: KNOWLEDGE AND VALUE ASSIGNED BY NURSING TEAM}

\begin{abstract}
Quantitative, descriptive and exploratory research which aimed to unfold the knowledge, the adherence and the value assigned by the nursing team of a renal diseases clinics about the central venous dressing in patients that were submitted to hemodialysis. Data were collected through a non participant systematic observation and individual interview. Analysis was conducted from the dimensions of the health beliefs model point of view. Results pointed out that the subjects assign value to the susceptibility of the patient in use of central venous catheter and in hemodialysis treatment to the development of infection as well as to the severity of the harms. They recognize the benefits of an assistance practice with quality to its prevention. Gaps between knowledge and adherence to the protocols were observed, and the adverse structural conditions were identified as the main barriers. Although workers know and assign value to the institutional protocol of dressing, by recognizing its importance in the infection prevention, there is a need of administrative and educational strategies for a closer relationship between theory and practice.
\end{abstract}

KEYWORDS: Catheterization, central venous; Bandages; Hemodialysis.

\section{CURATIVO DE CATÉTER VENOSO CENTRALEN PACIENTES RENALES: CONOCIMIENTO Y VALOR ATRIBUIDO POR ELEQUIPO DE ENFERMERÍA}

RESUMEN: Investigación cuantitativa, descriptiva y exploratoria que tuvo por objetivo investigar el conocimiento, la adhesión y el valor atribuido por el equipo de Enfermería de una clínica de enfermedades renales al protocolo de curativo de catéter venoso central en pacientes con hemodiálisis. Los datos fueron colectados por observación sistemática no participante y entrevista individual. El análisis fue direccionado por las dimensiones del modelo de creencias en salud. Los resultados evidenciaron que los sujetos atribuyen valor a la susceptibilidad de los pacientes renales usuarios de catéter venoso central y en tratamiento hemodialítico a desarrollar infección bien como la severidad de esos agravantes. Reconocen los beneficios de la práctica asistencial de cualidad para su prevención. Lagunas entre el conocimiento y la adhesión al protocolo fueron observadas siendo las condiciones estructurales desfavorables identificadas como principales barreras. A pesar de los trabajadores conozcan y atribuyan valor al protocolo institucional de curativo, reconociendo su importancia en la prevención de infecciones, son necesarias estrategias administrativas y educacionales para mayor aproximación entre la teoría y la práctica asistencial.

PALABRAS CLAVE: Cateterismo venoso central; Vendajes; Hemodiálisis.

${ }^{1}$ Enfermeira. Docente substituta da Universidade Federal do Paraná-UFPR.

${ }^{2}$ Enfermeira. Doutora. Docente Adjunto do Departamento de Enfermagem e Programa de Pós-Graduação em Enfermagem - Mestrado da UFPR. Membro do Grupo de Estudos Multiprofissional em Saúde do Adulto-GEMSA-UFPR.

Autor correspondente:

Elaine Drehmer de Almeida Cruz

Rua Pe. Camargo, 120 - 80060-240 - Curitiba-PR

Recebido: 03/03/09

Email: elainedrehmer@yahoo.com.br

Aprovado: 15/08/09

Cogitare Enferm 2009 Jul/Set; 14(3):470-5 


\section{INTRODUÇÃO}

Em tempos modernos as doenças crônicas ocupam espaço cada vez maior na assistência em saúde e, entre essas, a insuficiência renal crônica-IRC que consiste na perda brusca ou gradativa da função renal, na maioria das vezes associada às outras doenças, como Diabetes Mellitus e hipertensão $\operatorname{arterial}^{(1)}$.

Pessoas com IRC dependem de tratamento contínuo, como a hemodiálise, que imprime mudanças na rotina de suas vidas e ainda dependem de acesso venoso initerruptamente, ideal por meio de fístula arteriovenosa-FAV ${ }^{(1-2)}$. Porém, é comum ao início da hemodiálise a obtenção de acesso por meio da inserção de um cateter venoso central CVC de duplo lúmen em veia jugular, femoral ou subclávia( ${ }^{(2)}$, até a confecção e maturação da FAV. Embora seja um acesso temporário, o CVC imprime risco de complicações, sendo a mais relevante a infecção no sítio de inserção ou sistêmica, devido à quebra da integridade cutânea e tissular, permanência de um corpo estranho no espaço intravascular com manipulação frequente ${ }^{(3)}$.

As infecções associadas a CVC têm como fatores de risco as condições clínicas desfavoráveis, mas principalmente o tipo e localização do cateter, a frequência, a competência e a qualidade asséptica na manipulação ${ }^{(4,5)}$. A equipe de enfermagem tem papel fundamental na prevenção desses agravos, os quais correspondem ao aumento das taxas de morbimortalidade em pacientes submetidos ao tratamento hemodialítico ${ }^{(4,6-7)}$ porquanto, o cuidado qualificado e asséptico na manutenção deste dispositivo e a detecção precoce de sinais e sintomas preditivos de infecção contribuem para sua prevenção e limitação.

Neste contexto, destacam-se como oportunidades de cuidado a conexão e desconexão do paciente à máquina de hemodiálise e a realização do curativo de CVC, os quais devem ocorrer como rotina três vezes na semana e, em sua maioria, realizados por profissionais de enfermagem ${ }^{(8)}$. Nessas oportunidades, considera-se de vital importância na prevenção de infecções, a higienização das mãos, a desinfecção das conexões, a realização do curativo com material estéril e técnica asséptica, além da qualificação e treinamento do profissional de saúde $\mathrm{e}^{(3,9)}$.

Com vistas a padronizar a prática segura na prevenção de agravos, os serviços de saúde estabelecem protocolos específicos, porém é relevante a monitoração de seu uso pela equipe. Contudo, para que haja adesão, é necessário que os profissionais conheçam o protocolo, sejam capacitados e adquiram competência técnica e científica. Um fator pouco explorado é a investigação das possíveis razões da não-adesão ao determinado no protocolo, se essas devem-se ou não a falhas de conhecimento e/o valor atribuído às recomendações do mesmo.

Um dos métodos disponíveis para efetuar a análise de valor atribuído é o Modelo de Crenças em Saúde, desenvolvido da década de 50 por um grupo de psicólogos do Serviço de Saúde Pública dos EUA e que passou a ser uma alternativa aos que atuam em pesquisas ou intervenções na área de saúde ${ }^{(10)}$. Este método foi originalmente composto por quatro variáveis que podem influenciar a percepção de doenças ou comportamentos que levem à prevenção das mesmas, são elas: susceptibilidade percebida, severidade percebida, benefícios percebidos e barreiras percebidas.

A suscetibilidade percebida refere-se à percepção de riscos à saúde como, por exemplo, contrair infecção; a severidade percebida diz respeito à severidade ou gravidade da doença, como as consequências de uma infecção; os benefícios percebidos são relacionados às crenças na efetividade das ações para a saúde e suas consequências positivas; barreiras percebidas referem-se aos aspectos negativos, como gasto de tempo, custo-benefício, esforço, aborrecimento ${ }^{(11)}$.

Desenvolveu-se este estudo com o objetivo de investigar o conhecimento, a adesão e o valor atribuído pela equipe de enfermagem ao protocolo de curativo de CVC de um serviço de hemodiálise.

\section{MÉTODOS}

Trata-se de uma pesquisa de campo, descritiva exploratória e de abordagem quantitativa, realizada de acordo com a Resolução CNS n. 196/96, no período de agosto a novembro de 2008, após aprovação pelo Comitê de Ética em Pesquisa sob registro CEP/SD 559.096.08.06 CAAE1774.0.000.091-08.

Participaram da pesquisa oito profissionais da equipe de enfermagem do período matutino, entre os 12 atuantes, de uma clínica especializada em tratamento de doenças renais do Estado do Paraná e que manipulam e realizam curativo de CVC em pacientes renais crônicos durante as sessões de hemodiálise.

Para a coleta de dados foram utilizadas duas estratégias. A primeira estratégia foi a realização de 
entrevista individual com preenchimento de um instrumento, com perguntas abertas e fechadas, elaborado para este estudo com base no protocolo institucional para a realização de curativo de CVC, nos princípios de prevenção de infecções associadas a $\mathrm{CVC}^{(3)}$ e nas dimensões de crenças em saúde de Rosenstock $^{(12)}$. A segunda estratégia foi a observação não participante sistematizada da adesão ao protocolo de curativo de CVC e das barreiras à adesão deste, usando instrumento elaborado com base no protocolo institucional contendo uma lista de verificação para o preenchimento do campo sim ou não bem como um campo para a anotação das observações do pesquisador.

Os dados objetivos de ambos os instrumentos alimentaram um banco de dados do programa Excel (versão 2007). Os dados subjetivos, obtidos a partir das entrevistas e das observações do pesquisador, foram listados segundo a freqüência e agrupados por similaridades em categorias, de acordo com a técnica de análise do discurso ${ }^{(13)}$. A análise e discussão dos dados foi direcionada pelas dimensões do Modelo de Crenças em Saúde ${ }^{(12)}$ : suscetibilidade, severidade, benefícios e barreiras percebidas, a considerar que podem influenciar a percepção ou comportamentos. Ainda utilizou-se princípios de prevenção de infecções associadas a $\mathrm{CVC}^{(3)}$ e do protocolo institucional para a análise e discussão dos resultados.

\section{RESULTADOS E DISCUSSÃO}

Participaram deste estudo um enfermeiro, um auxiliar e seis técnicos em enfermagem; o tempo médio de atuação em saúde era de 137 meses (aproximadamente 11 anos) e o de atuação na clínica de nefrologia de 63 meses (aproximadamente 5 anos).

Considerando o protocolo institucional para a realização de curativo de CVC no que diz respeito ao uniforme, apresentação pessoal e uso de adornos observou-se que a totalidade dos sujeitos usava cabelos presos ou curtos sob touca, uniforme próprio, calçados fechados e limpos. Um profissional usava esmalte e todos tinham as unhas aparadas e limpas. Seis (75\%) trabalhadores usavam adornos como anel, aliança, relógio, brincos e suporte pendente para crachá.

Um conjunto de recomendações para trabalhadores de serviços de saúde, com destaque à higienização de mãos-HM, manutenção de unhas curtas e sem esmalte, ausência de adornos e uso de equipamentos de proteção individual-EPI, incluindo o uniforme, visa a segurança de pacientes e profissionais.
Apesar de normas nacionais ${ }^{(14)}$ e internacionais ${ }^{(15-16)}$ amplamente divulgadas no meio acadêmico e da saúde, várias pesquisas evidenciam lacunas entre o recomendado e a prática, especialmente quando investigada a adesão à $\mathrm{HM}^{(17)} \mathrm{e}$ o uso de $\mathrm{EPI}^{(18-19)}$, agravando os riscos e expondo usuários e trabalhadores ${ }^{(20)}$.

Neste estudo todos os sujeitos consideram a HM de extrema importância para a prevenção de infecções, porém somente cinco $(62,5 \%)$ referem realizá-la sempre que necessário, ou seja, antes e após cada procedimento. Na observação sistemática foi constatada esta prática inadequada, com falhas na HM após a realização do curativo e retirada de luvas. Considerando-se que o protocolo institucional preconiza o uso de luvas para a realização do curativo, esse resultado vem ao encontro de estudo que demonstrou ser o uso de luvas um preditor para a não adesão à HM pela falsa sensação de segurança integral ${ }^{(21)}$. Deve-se considerar que no local da pesquisa há estrutura necessária à HM, como pias, torneiras, sabão, papel toalha e álcool 70\%, o que viabiliza esta prática. Apesar de todos valorizarem no discurso a HM como medida de prevenção de infecções, essa por vezes é negligenciada por alguns deles. Esse resultado evidencia um descompasso entre o conhecimento, as crenças e a atitude dos trabalhadores em saúde ${ }^{(22)}$.

Para HM eficaz é necessária a fricção de toda a superfície das mãos e dos punhos, inclusive dos espaços interdigitais, utilizando-se álcool ou sabonete líquido com ou sem anti-séptico ${ }^{(23-24)}$. As mãos higienizadas contribuem para a prevenção de infecções, sendo relevante mantê-las saudáveis, sem adornos, mantendo unhas curtas e sem esmalte ${ }^{(15,25)}$, considerando-se que sob as unhas e adornos evidenciam-se altas contagens bacterianas ${ }^{(20)}$.

Ao investigar a competência e o conhecimento para atuar em hemodiálise e acerca do protocolo de curativo de CVC, seis sujeitos (75\%) referiram ter recebido capacitação para atuar em hemodiálise, sete (87,5\%) participaram das atividades de educação em saúde promovidas pela instituição, porém dois (37,5\%) tinham dúvidas quanto à assistência de enfermagem em hemodiálise. Todos conceituaram infecção corretamente e a referiram como a principal complicação associada ao tratamento; seis profissionais (75\%) consideraram como Alto o risco em adquirir infecção associada a CVC e dois (25\%) o consideraram como Médio. Como agravos desses eventos adversos foram citados o óbito, a necessidade 
de troca do cateter e seu impacto negativo na qualidade de vida dos pacientes. A totalidade dos sujeitos percebe o curativo como elemento essencial na prevenção de infecções e apenas um referiu ser elemento de redução de custos.

Em resposta à pergunta: Cite cuidados essenciais na realização do curativo na prevenção de complicações, todos referiram a observação do protocolo institucional e a manutenção de técnicas assépticas, citando como exemplos a HM, desinfecção das conexões entre equipo e cateter antes da desconexão, uso de material de curativo estéril e manutenção de um ambiente seguro para a realização do curativo de CVC. Este ambiente foi mencionado como aquele mais limpo possível, com estrutura que permita a realização da técnica de forma adequada, como ausência de pessoas estranhas e não realização de limpeza ambiental durante o procedimento, além de condições de estrutura física e material.

Quando investigados os benefícios na adoção da técnica correta de realização de curativo de CVC, sete $(87,5 \%)$ concordaram totalmente que esta prática evita infecções. Reconheceram também a contribuição para a qualidade de vida, menor ocorrência de óbitos prematuros e manutenção da vida útil do cateter. Esses resultam em benefícios para a equipe por prevenir o mau funcionamento do cateter, reduzir gastos, promover melhor aproveitamento do tempo e maior satisfação do empregador, segundo os sujeitos.

Quanto às barreiras percebidas para a realização do curativo de acordo com protocolo, três $(37,5 \%)$ citaram a falta de mesa auxiliar, o que para eles, além de inadequado esteticamente, dificulta a realização do curativo e prejudica a observação da técnica asséptica, uma vez que material é colocado no colo do paciente. O número insuficiente de profissionais, segundo os sujeitos da pesquisa, faz com que o trabalho tenha que ser realizado com maior rapidez, expondo ao risco ocupacional e ao risco de infecção. Outras barreiras percebidas foram a falta de material em quantidade suficiente e deficiências no conhecimento científico.

Os resultados da observação sistematizada da adesão ao protocolo e de barreiras na prática assistencial reiteram o discurso dos sujeitos quanto à disposição incorreta do material estéril no momento do curativo, por falta de mesa de apoio; improvisação da técnica por falta de material e não HM frente a todas as oportunidades.

Também foi observada a contaminação dos conectores durante a sua manipulação. A ação de conectar e desconectar os protetores do cateter duas a três vezes por semana, para as sessões de hemodiálise, é reconhecida como fator de risco para infecção; a inobservância das técnicas assépticas potencializam esse risco ${ }^{(8,26)}$. A comunicação com o paciente, a inspeção e o registro de sinais preditores de infecção e das condições clínicas do paciente são práticas recomendadas no protocolo e realizadas pela equipe investigada, bem como os passos para a limpeza e anti-sepsia do sítio de inserção do CVC e equipo, além da heparinização do cateter.

A educação continuada, a capacitação da equipe e a elaboração de protocolos para a prevenção e controle de agravos são elementares à prevenção das infecções, porém urge avaliar, continuamente, o sincronismo entre a teoria e a prática assistencial, pois reflete na qualidade dos serviços. Deste modo, também as condições de trabalho, os recursos materiais e serviços de apoio são relevantes neste contexto ${ }^{(27)}$. A educação da equipe de saúde é apontada como a medida mais importante para prevenção das complicações referentes ao uso de CVC e a HM é medida primordial para prevenção de infecções ${ }^{(28)}$.

Cabe aos profissionais de saúde compartilhar a responsabilidade de ofertar ao paciente um ambiente seguro, em busca da ausência de riscos. Entre esses, as infecções são prejudiciais tanto para o paciente quanto para o profissional, seja no aspecto clínico como financeiro, visto que, além do sofrimento e gastos excessivos com medicamentos, geram processos judiciais ${ }^{(20)}$. A coerência entre os protocolos institucionais, o contexto assistencial e a prática profissional contribui para a prevenção de infecções associadas à assistência à saúde.

\section{CONSIDERAÇÕES FINAIS}

A terapêutica renal substitutiva é fundamental em caso de insuficiência renal. A perda do acesso vascular por infecção associada a CVC imprime risco de morte, aumento de custos e prejuízo para a rotina de vida do paciente, já alterada pelo tratamento. A equipe de enfermagem tem papel importante na prevenção desses agravos e os protocolos buscam nortear e padronizar técnicas corretas, havendo a necessidade do conhecimento, viabilidade operacional e adesão a esses.

Os resultados desta pesquisa apontam que os profissionais de enfermagem reconhecem as 
consequências do cuidado direcionado ao paciente em hemodiálise e com CVC; têm conhecimento e valorizam as medidas de prevenção de infecção associadas a esses dispositivos, constantes do protocolo institucional. Porém, barreiras estruturais e falta de adesão a elementos fundamentais à prevenção dos agravos são desafios a serem enfrentados. Entendemos que esta pesquisa contribuiu para a qualidade da assistência a paciente em hemodiálise, por realizar um diagnóstico situacional e potencialmente direcionar a busca de soluções para os fatores desfavoráveis identificados.

\section{REFERÊNCIAS}

1. Fermi MRV. Manual de diálise para enfermagem. Rio de Janeiro: Medsi; 2003.

2. Arone EM, Philippi MLS. Enfermagem médico-cirúrgica aplicada ao sistema renal e urinário. $2^{\mathrm{a}}$ ed. São Paulo: Senac; 2005.

3. O’Grady NP, Alexander M, Dellinger EP, Gerberding JL, Heard SO, Maki DG, Masur H, McCormick RD, et al. Guidelines for the prevention of intravascular catheterrelated infections. Centers for Disease Control. Morb Mortal Wkly Rep. 2002;51(RR-10):1-26.

4. Lima EM, Martinho GH. Infecções relacionadas à Unidade de Hemodiálise. In: Oliveira AC. Infecções hospitalares: epidemia, prevenção e controle. Rio de Janeiro: Guanabara Koogan; 2005. p. 577-92.

5. Ferreira V, Andrade D. Cateter para hemodiálise: retrato de uma realidade. Medicina 2007;40(4):582-8.

6. Nettina SM. Prática de enfermagem. $7^{\mathrm{a}}$ ed. Rio de Janeiro: Guanabara Koogan; 2003. Distúrbios renais e urinários. p. 503-552.

7. Bonvento M. Acessos vasculares e infecção relacionada à cateter. Rev Bras Terap Intensiva 2007;19(2):227-30.

8. Ferreira V, Andrade D, Santos CB, Moysés Neto M. Infecção em pacientes com cateter temporário duplolúmen para hemodiálise. Rev Panam Infectol 2005;7(2):16-21.

9. CDC - Centers For Disease Control And Prevention. Department of Health and Human Services. Intravascular device-related infections prevention; guidelines availability; notice. Part II. Atlanta: CDC, 1995.
10. Melo DS. Adesão dos enfermeiros às precauções padrão à luz do modelo de crenças em saúde [dissertação]. Goiânia: GO. Faculdade de Enfermagem/Universidade Federal de Goiás; 2005. 191 p.

11. Dela Coleta MF. Escalas para medida das crenças em saúde: construção e validação. Aval Psicol 2003;2(2):1112.

12. Rosenstock IM. The health belief model and preventive health behavior. Health Educ Monogr 1974;2(4):304-87.

13. Bardin L. Análise de conteúdo. Lisboa: Edições 70; 1977.

14. Ministério da Saúde (BR). Agência Nacional de Vigilância Sanitária. Higienização das mãos. Brasília; 2008. Disponível: www.anvisa.gov.br/servicosaude/ controle/publicacoes.htm

15. Bоyce JM, Pittet D. Guideline for hand hygiene in health-care settings: recommendations of the Healthcare Infection Control Practices Advisory Committee and HICPAC/SHEA/APIC/IDSA Hand Hygiene Task Force. Morb Mortal Wkly Rep. 2002;51:1-45.

16. WHO. Guidelines on hand hygiene in health care. First global patient safety challenge clean care is safer care. Geneva, 2009.

17. Cruz EDA, Pimenta FC, Palos MAP, Silva SRM, Gir E. Higienização de mãos: 20 anos de divergências entre a prática e o idealizado. Cienc Enferm. 2009;15(1):33-8

18. Brevidelli MM. Exposição ocupacional ao vírus da AIDS e da hepatite B: análise da influência das crenças em saúde sobre a prática de reencapar agulhas. [dissertação]. São Paulo (SP): Escola de Enfermagem, Universidade de São Paulo; 1999.

19. Sarquis LMM, Felli VEA, Miranda FMD, Guimarães HV, Oliveira GP. A adesão ao protocolo de monitoramento dos trabalhadores de Saúde após exposição a fluidos biológicos: uma problemática vivenciada em um ambulatório de saúde do trabalhador no Paraná. Cogitare Enferm. 2005;10(2):47-53.

20. Bolick D, Brady C, Bruner DW, Edelstein S, Lane K, McLaughlin MB et al. Segurança e controle de infecção. Rio de Janeiro: Reichmann \& Affonso; 2000.

21. Girou E, Chai SHT, Oppein F, Legrand P, Ducellier D, Cizeau F, Brun-Buisson C. Misuse of gloves: the foundation for poor compliance with hand hygiene and potential for microbial transmission? J Hosp Infect. 2004;57:162-9. 
22. Creedon SA. Healthcare workers' hand decontamination practices: compliance with recommended guidelines. J Adv Nurs. 2005;51(3):208-16.

23. Martini AC, Dall'agnol CM. Por que lavar as mãos? Motivos de um grupo de enfermagem. Rev Gaucha Enferm. 2005; 26(1): 88-101.

24. Fernandes AT, Fernandes MOV. Organização e programa de controle das infecções hospitalares. In: Fernandes AT, Fernandes MOV, Ribeiro Filho N. Infecção hospitalar e suas interfaces na área da saúde. São Paulo: Atheneu; 2000. p.1461-81.

25. Ministério do Trabalho e Emprego (BR). Legislação. Norma regulamentadora $n^{0} 32$. segurança e saúde no trabalho em estabelecimentos de saúde. Portaria GM n. ${ }^{\circ}$ 939, de 18 de novembro de 2008. Brasília; 2008. Disponível: www.mte.gov.br/legislacao/normas_regula mentadoras/default.asp

26. Oliveira SCV, Steckert JS, Russi RF, Steckert Filho A. Cateteres totalmente implantáveis em pacientes oncológicos: análise de 178 casos. Arq Cat Med. 2008;37(1):43-8.

27. Nonino EAPM, Anselmi ML, Dalmas JC. Avaliação da qualidade do procedimento curativo em pacientes internados em um hospital universitário. Rev Latino-Am Enferm. [periódico na Internet]. 2008 Fev [acesso em 2009 Mai 08]; 16(1): 57-63. Disponível: www.scielo.br/ scielo.php?script=sci_arttext\&pid=S0104-116920080001 00010\&lng $=\mathrm{pt}$

28. Mesiano E, Merchán-Hamann E. Infecção da corrente sanguínea em pacientes em uso de cateter venoso central em Unidades de Terapia Intensiva. Rev LatinoAm Enferm. 2007;15(3):453-9. 